\title{
The Reform in Knowledge Service in the Internet Age
}

\author{
Xueyi Duan \\ School of Economics and Management, Beijing University of Posts and Telecommunications, Beijing, 100876, \\ China \\ 18301331066@163.com
}

Keywords: Knowledge service; product form; pricing model; management model.

\begin{abstract}
With the development of internet technology, the age of the knowledge economy has come and brought about the substantial reform in knowledge service, which prompted platform vendors and users to adjust their behavior in accordance with different kinds of reforms. To learn about the change of knowledge service in all aspects, this paper conducted statistical analysis to explore modern Internet-based knowledge service platforms from multiple angles. After in-depth investigation and case analysis, the author found that the form of knowledge service platforms had been verifying, innovative products had been springing up and the rights of pricing had been gradually transferring from merchants to consumers. In this case, a huge challenge was posed to knowledge-based enterprises regarding to their management and marketing models and they began engaging themselves in various new attempts, strengthening the application and innovation of existing theories, drawing on the experience of other successful enterprises and consolidating each link in management and marketing in order to survive in the ever-changing market.
\end{abstract}

\section{Introduction}

By June 2017, the number of netizen in China has added up to 751,000,000 and the penetration of Internet has reached 54.3\%, increasing by 1.1\% compared to that in the end of 2016 and showing a trend of steady year-by-year growth. The majority of the netizens is the group aged from 10 to 39 (account for about 72.1\%), which covers mostly students, freelancers and enterprise employees. Above-mentioned data fully demonstrates the healthy development of domestic Internet industry, the stability of domestic netizen structure and the enormous potential of domestic markets.

In terms of business service, adaptations were made in light of the netizen structure mainly consisting of the young group. The emerging knowledge services captured the attention of numerous netizens and the user scale achieved a significant growth. By October 2017, the number of monthly active users of typical knowledge product-based applications is shown in the table 1.

Table 1 Number of Monthly Active Users of Typical Knowledge

\begin{tabular}{|c|c|c|}
\hline APP & Launch Date & $\begin{array}{c}\text { Number of Monthly Active } \\
\text { Users }\end{array}$ \\
\hline Himalaya FM & 2013.3 & 3566.53 \\
\hline Zhihu & 2010.12 & 1371.3 \\
\hline Huajiao Live-Stream & 2015.6 & 1133.5 \\
\hline Douban.com & 2005.3 & 469.11 \\
\hline Iget & 2016.5 & 236.56 \\
\hline Fenda & 2016.5 & 9.92 \\
\hline
\end{tabular}

Unit: 10,000 persons

(Data from: White Paper on the Development of China's Knowledge Payment Industry)

Therefore, it can be inferred that consumers and users pay more attention to the "sale" of knowledge and culture along with the wave of Internet-based innovation and entrepreneurship and people's increasing requirement for the quality of spiritual life. In addition, applications which 
provide Q \& A of encyclopedic knowledge, resources of professional papers, original viewpoints and thinking, channels of language learning and leisure and entertainment services etc emerge in endlessly and take a substantial market share. These services play an important role in improving domestic people's cultural quality and spiritual realm by offering netizens positive and healthy ways of entertainment and imparting them knowledge and original thoughts in a more affordable, convenient and environmental way. Additionally, these newly emerged services have caused a huge change in the product form and pricing way of China's Internet industry and posed a considerable challenge to the enterprises that mainly provide knowledge services.

\section{The Diversification of Product Form}

Knowledge economy era is witnessing the enrichment of the knowledge product form. Most of the traditional knowledge services take such forms as paper media, publication and off-line classroom and impose a stricter requirement on the professionalism of imparters. Meanwhile, the receivers are required to have a sound economic basis and knowledge reserve. As regards paper media and publication, they seem more like spreading rather than exchanging of knowledge for the lack of interaction. Due to the development of Internet technology and the popularity of applications, current knowledge services break away from conventions, keep adopting innovative modes (as shown in chart 2) and thus affording a wider scope of knowledge. For example, HowNet can efficiently integrate a huge mass of paper resources and built multiple index mechanisms like references with the use of big data; the production of knowledge tends to popularize that every user can convey their views and thoughts to the masses through platforms like WeChat public account and Sina Weibo at a low cost; the exchange of knowledge become highly popular that applications like Zhihu offers users from different fields and with different needs plenty of opportunities to share their learning experience and have lengthy discussions on all sorts of topics; knowledge spreads in a more diverse way due to the development and combination of medium technology, from the characters at the very beginning to the audio medium for learning languages and listening to music, the videos of online education, television and film, and the extremely popular network broadcast nowadays.

Table 2 Categories of Typical Emerging Knowledge Products

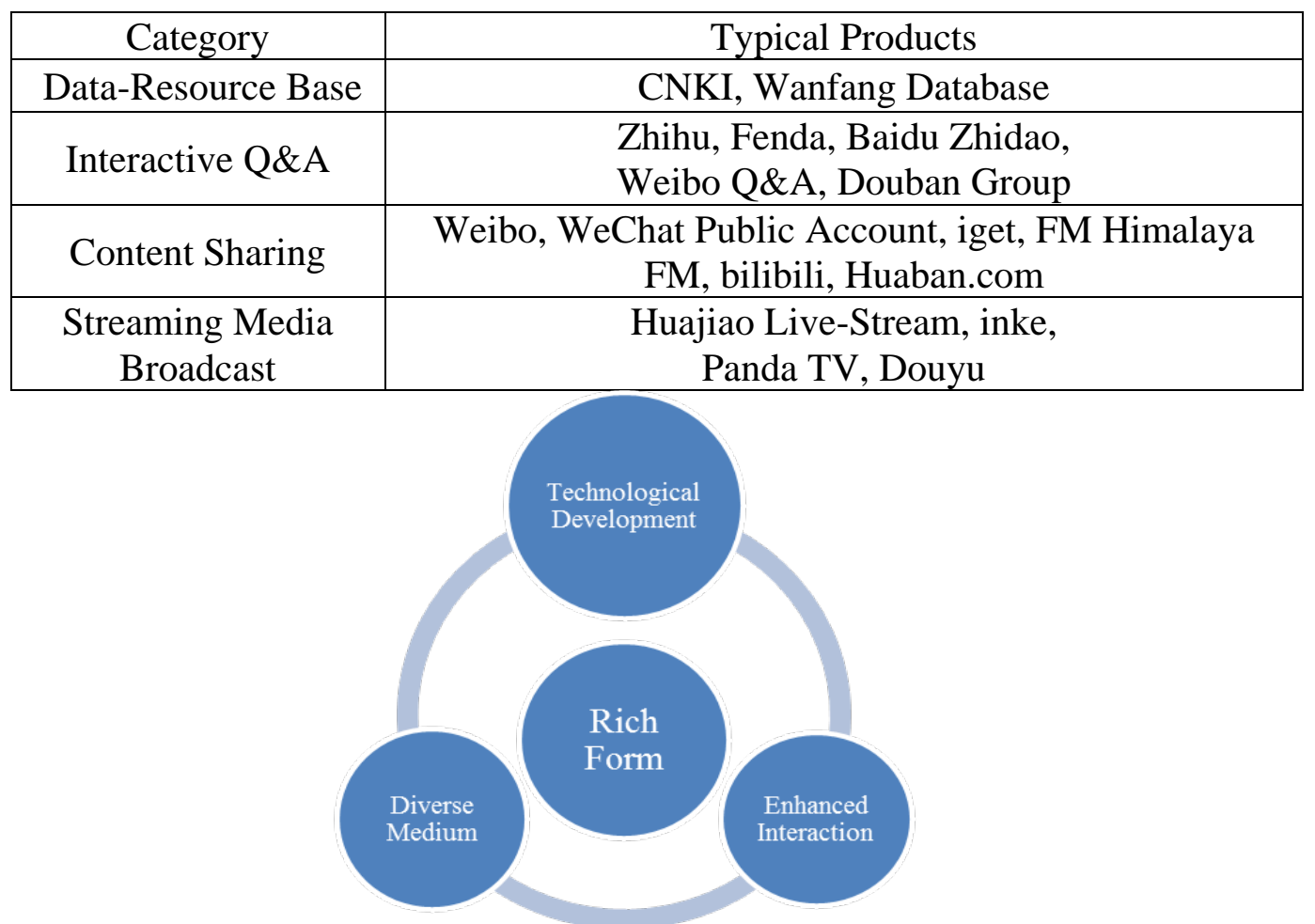

Based on the original connotation of knowledge, the form of product appeared to be more interesting and creative and user experience was further optimized. Moreover, knowledge-based 
products kept springing up and captured more and more consumers' attention. In this way, knowledge service gradually turned into a significant branch of the Internet industry. Some products were directly aimed at a majority of consumers and a series of technologies were applied to offer higher-quality services widely accepted by the audiences from different dimensions, including amount, quality and speed; yet, some products were designed for only a few or even a single consumer without duplication and batch production, but they could exactly serve corresponding consumers' personal needs and thus maximized the consumer utility.

It is indicated by above-mentioned situation that the knowledge industry is being divided more complexly from a different angle and the connection and cooperation between upstream and downstream becomes closer and they may even integrate with each other. In the "The Knowledge-Based Economy" released by the Organization for Economic Co-operation and Development in 1996, it is considered that knowledge mainly depends on the technology incorporated in it, namely the nature and essence of knowledge itself, thereby knowledge is divided according to the technology applied into high technology, intermediate technology and low technology. In China, the knowledge industry includes the industry of knowledge production (like scientific research institutions) and the industry of knowledge diffusion (like publishing institutions) in light of the three-time industry division at home. Differently, the traditional division can no longer satisfy the requirements of modern knowledge industry. Nowadays, the division bases more on the category of service and the medium of knowledge diffusion, and every ordinary individual can be the knowledge producer and an independent enterprise can build a complete system of knowledge service and provide a platform with all-round services via multiple media. In this case, both the production and diffusion of knowledge is included in one process, the boundary between them is not clear any longer, and different technologies are employed to meet diverse demands.

\section{The Beginning of Pricing Rights Transference}

As a trading object, knowledge inevitably bears a price of its own. Different from traditional commodities which are priced by sellers, knowledge services are priced according to their specific forms and contents, principally in three different ways, which jointly embody the transfer of pricing rights. The first one is that the price is completely set by platforms and HowNet is a case in point. By taking advantage of its rich resources, HowNet gains a monopoly as seller. For ordinary consumers, prices are set by the seller in accordance with the articles they download; for institutions like colleges and universities, the seller will consider the quality and quantity of their literature and then negotiate with different institutions to set corresponding prices, which is regarded as complete price discrimination. The second one is that the price is set by knowledge providers and platforms charge small intermediary fees. Weibo Q \& A and Zhihu, as a typical example, still differ from one another in the distribution of residual income as the former one is shared by quizzers and answerers while the latter one is entirely possessed by answerers. The third one is that the price is totally set by knowledge demanders, and the tipping behavior on network broadcast platform and WeChat public account is a representative example. Audiences (or readers) willingly pay a certain amount of money for their favorable impression on or recognition of the contents of broadcast or articles, and this is all decided by consumers' personal utility and seemed as maximized benefit for consumers.

With the continuous improvement of material life, an increasing number of people pay more attention to their spiritual consumption and place more emphasis on the importance of knowledge products. Correspondingly, knowledge products are becoming privatized and individualized and the scale economies effect of the production of knowledge products is lessening day by day. Additionally, consumers are willing to pay a higher price due to the increasing welfare for them. In today's knowledge service industry, the second and the third kind of way of charging are widely adopted, especially the third one; in the meantime, the number of application platforms is increasing day by day and they are becoming the mainstream step by step, which means the rights of pricing knowledge products is being transferred to consumers.

In this course, a typical behavior was generated and known as tipping, which means that 
consumers can pay for knowledge services according to their satisfaction in the way of cash or virtual presents. As the tipping behavior nearly allows consumers to have the pricing rights, the platforms are motivated to carry out rigorous examination and improve the quality of their products and services. Driven by the network economic effect and consumers' herd mentality, some service could attract more people to follow and tip after it achieved huge popularity among the public or won the comment and praise of certain celebrity, thereby forming the Internet celebrity economy and fan economy. It is shown by relevant researches that as for platforms, consumers appear to tip most on Q\&A platforms like Zhihu, which accounts for 37.81\%; as regards contents, intellectual contents take a major share and live broadcast boasts tremendous market potential in spite of its small share. The distribution of tipping objects is displayed as follows:
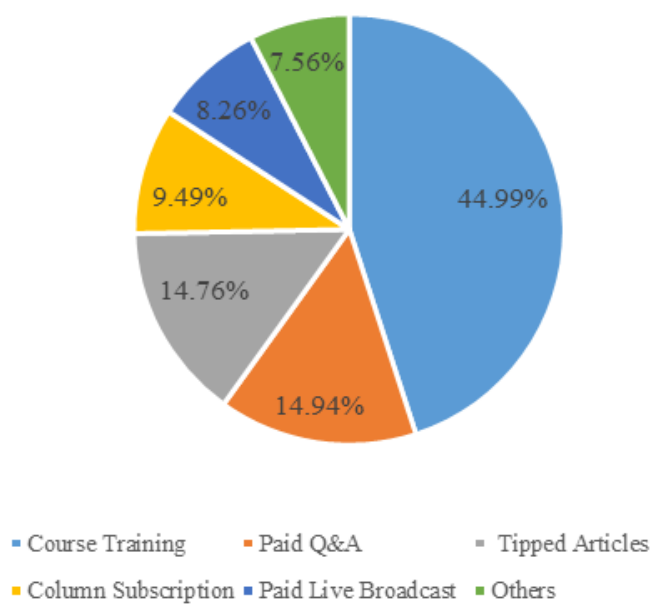

Fig 1 Distribution of Tipping Objects

Course training, obviously knowledge-oriented, is a prior combination of Internet technology and traditional industry; topic Q\&A mostly centers on the knowledge of certain subject and it also revolves around certain phenomenon, while articles on public accounts generally convey their original thoughts and opinions on hot social issues to the public, both of which account for more than half of the contents tipped. Hence, it is more acceptable to express knowledge and profound thoughts in a leisurely way. As a strong contrast, live broadcast tipping seems more like consumers' amusement and entertainment. Live broadcasting, with its contents focused on stars' or network celebrities' daily life or certain part of their routine work, interacts with the audience by answering their questions or fulfilling their particular requirements. The audience buys virtual currency or presents on the platform for the hosts they like and the income is shared by platform owners and hosts. The pattern is presented in the graph below. Besides, the income of the live broadcast industry is on the rise and as an emerging sector of knowledge industry, it is developing at an astonishing speed and this gives the masses every reason to believe that the potential of it is huge, the cake will be bigger and it will be a significant whirlwind in the future. Among the bunch of popular live broadcast platforms, Huajiao, inke and MOMO are typical examples. According to "Annual Big Data on Live Broadcast of Huajiao Live Stream”, the earnings of Huajiao's hosts in 2016 added up to over RMB $5,000,000.00$, which is really amazing as far as profitability is concerned. 


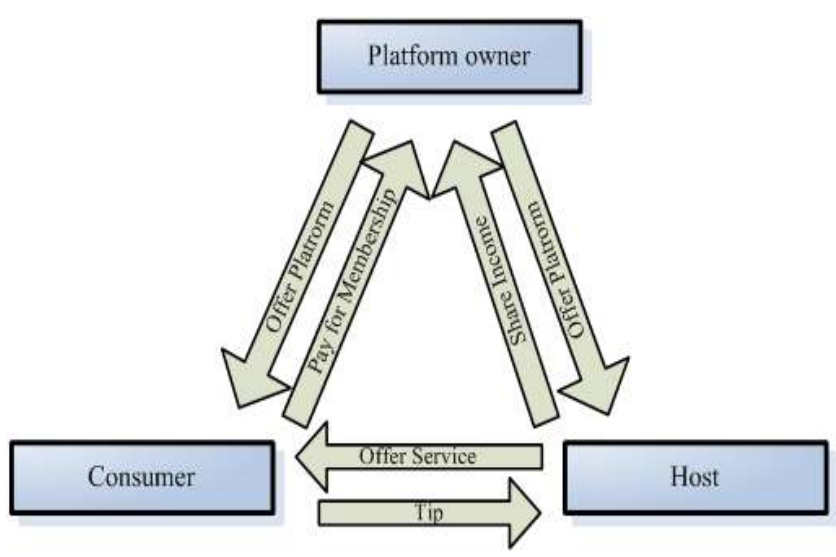

The transference of pricing rights not only directs the development of knowledge service pricing model, but also reflects the new trend of product pricing in the future. On the one hand, the commercialization of knowledge and the heightened awareness of pricing knowledge products indicate Chinese citizens' increasing awareness of intellectual property protection, active learning, energetic pursuit of higher spiritual realm, growing respect for knowledge, knowledge workers and their fruits of labor, improving intellectual level and legal cognition. On the other hand, the information asymmetry between buyer and sellers began to reduce, the consumers are no longer in a weak position in trades, and the prices are decided more by the relation between supply and demand. Therefore, the market imposes stricter requirements on the quality of products and it is of great significance for enterprises to consider how to attract consumers, promote consumer utility and thus cultivate "regular customers"

\section{The Challenge to Enterprises Providing Knowledge Products}

It can be known from the analyses mentioned above that the innovation of knowledge products keeps going on in a variety of ways, more and more new enterprises are breaking into the market and thereby the competition is strengthened; the pricing rights began to transfer to consumers, merchants cannot enjoy their initiative any more, the realization on earning becomes less predictable, and the absolute growth of revenue gets increasingly doubtful. To take upon these challenges, enterprises shall pay more attention to the adjustment of their management and marketing strategies.

\subsection{The Management Strategy of Enterprises Providing Knowledge Products}

Knowledge-intensive enterprises are mainly composed of knowledge workers, and aforementioned emerging enterprises which feature knowledge products, in particular, contain a majority of young, creative, passionate, challenge-embraced and well-educated employees who look forward to novel things and interesting experiences. However, the conventional management mode of traditional enterprises failed to stimulate these employees' productivity and creativity, so these enterprises are in an urgent need to thoroughly reform their management mode, defining the code of conduct with rigid systems and attracting more outstanding talents of the new age with flexible mechanisms.

First and foremost, enterprises shall establish comprehensive talent assessment mechanisms and employment standards and recruit new staff based on their hard skills and soft skills. Hard skills include the interviewees' technical and professional skills which can be fully presented in written examination, experiment and quiz; soft skills are reflected more by the interviewees' thinking mode and values in their speech and deportment. It is highly important for enterprises to find partners and co-workers who share their cultural concept instead of pure labor forces, namely finding "people of the same mind" and ensuring they will undertake every mission with professional skills, positive attitude and real passion.

Secondly, enterprises shall enhance the training for new employees in a bid to strengthen their organizational commitment and sense of pride as well as help them adapt to the enterprise's working pattern and pace. The training is supposed to focus on employees' hard and soft skills, take a novel 
and interesting form, and emphasize no more classes. Besides, the training should no longer be a simple introduction of work processes, rules and regulations but a long process of recording employees' growth and transformation from listening to speaking and from being passive to being active, generally heightening employees' sense of ownership and then increasing the new employees' activeness and participation. As every employee will have their own supervisor to instruct them in their work and career planning, enterprises shall sharpen the supervisors' sense of responsibility and require them to be responsible for their words and behaviors and their trainees. For example, in Huawei all staff can seek one-on-one assistance from their supervisors, and the company gives every supervisor certain allowance for the purpose of remaining their activeness and enthusiasm and facilitating the communication between supervisors and trainees. In addition, if trainees made mistakes at work, their supervisors will assume corresponding responsibility and receive certain punishment for the mechanism of joint responsibility.

Thirdly, incentives for employees are very important and the form, content and cycle of incentives also worth detailed exploration. Though direct material motivation can somehow stimulate employees' desire and determination to work hard, it may, in the long run, weaken employees' sense of pride for realizing their value, foster their pursuit of interests, and even build up their regular expectation for material motivation, which will result in employees' negative work attitude and enterprises' lower operating efficiency if they failed to obtain them. On the contrary, the lack of material motivation may prompt employees to feel confused and disappointed about their prospect in comparison with their peers in other enterprises and under the heavy life pressure. Consequently, the employees become less energetic but more negative at work and thus the production will be severely hindered. Therefore, enterprises need to comprehensively weigh the advantages and disadvantages of both measures and seek for an optimal solution so as to maximize the benefits at a low cost. For one thing, enterprises shall combine regular and irregular as well as quantitative and non-quantitative motivation, centering on irregular and non-quantitative motivation. Enterprises can create surprises for employees through irregular and non-quantitative motivation and thus bring them great happiness and allow them to feel the enterprise's constant attention to them. Also, employees will stay vigorous and passionate at work and hold an optimistic expectation of their future, and employees are encouraged to perfect their mind and work harder in order to achieve better performance and heighten their consciousness of innovation. For another thing, to integrate material and virtual motivation, the management of enterprises shall devise interesting and precise assessment mechanisms to stimulate all employees to actively strive for incentives and prevent any cut-throat competition. Hence, it is extremely important to go deep among the employees and learn about their hobbies and interests; meanwhile, enterprises shall motivate their employees to think from the standpoint of companies and devote themselves to the companies' sustainable development instead of merely improving their own performance. To that end, enterprises shall call upon employees of different departments to help each other and make progress together; on top of that, employees shall be given corresponding incentives as long as their measures and suggestions are conducive to the enterprise, so the form of motivation shall be further diversified. For example, the team of Luogic Show, which consists mainly of post-85s and successfully runs the iget APP, adopts a novel game-based motivation mode to foster their employees' activeness and participation and help them better grow. This team motivates their employees with "moral integrity coins" and they have no fixed assessment mechanism; instead, they deliver the rights of grading to other colleagues in order to heighten every employee's sense of participation, facilitate the interaction among employees, create a friendly and stimulating work environment, and truly and objectively reflect the employees' actual conditions. Employees can use their "moral integrity coins" to pay their bills in the company's cooperative restaurants, the quarterly or monthly "King of Moral Integrity" will receive different forms of material motivation, and the content of value of incentive remain uncertain in a bid to keep the gamification feature of virtual motivation tools and create continuous surprises. In addition to the incentives, the title also stands for honor and other colleagues' recognition and trust, which seems more like a kind of spiritual satisfaction and motivation.

The management philosophy of knowledge-intensive enterprises emphasizes people first and 
cares much about people's feelings. Enterprises are supposed to choose like-minded partners, actively accept the new employees, offer enough support to employees in need, concern about the process of their growth. At the meantime, enterprises shall help employees heighten their sense of responsibility, cultivate their sense of ownership, maintain their personality and concurrently adapt to the corporate culture, and offer them opportunities for practice and professional guidance, thus allowing them to get familiar with the operational process and sharpen their business skills. Moreover, enterprises shall innovate the ways of motivation, maintain employees' passion and prompt them to deeply feel the realization of their value and the recognition from others, so as to achieve a virtuous cycle.

\subsection{The Marketing Strategy of Enterprises Providing Knowledge Products}

In the face of increasingly diverse and personalized consumer demands, enterprises need to form their own culture and concept: if focusing on technology, more emphasis should be placed on the influence of products' connotation and the advancement and accuracy of contents; if focusing on feelings, the products should be designed and packaged in an eye-catching way; if aiming at the young group, products should be presented in vivid and interesting ways; if aiming at high-end users, products should be presented in a formal and rigorous way. In this way, enterprises can unify the style of their products, be it the form or content of them, and enjoy a clear recognition in the market. For example, different from those APPs which invited popular stars to be their spokesman, Zhihu has four influential celebrities with remarkable success in their own fields as its spokesman, including Audrey Hepburn, Hemingway, Picasso and Schrodinger, so as to correspond with its knowledge-advocating concept of "finding a bigger world".

As for product marketing, the marketing theory of 4Ps should be fully used for the formulation of innovative marketing strategies, and the company should seize the opportunities for development in the big data age. Enterprises shall segment the market, focus on the same knowledge and develop different innovative products in different forms for different consumers, deliver them to particular groups of users and thereby bolster the attraction and selling point of every product. Meanwhile, enterprises shall enrich their way of publicity by utilizing the features of the knowledge economy age and the Internet age, promote with the help of co-partners, media platforms and consumers, observe the audiences' interest and create hot topics to extend influence and achieve an increase in popularity. Also, enterprises shall reform their marketing channels, reduce intermediate links, find more ways to directly communicate with consumers, optimize services and enhance users' satisfaction. Furthermore, enterprises shall place emphasis on the quality of product and connotation of knowledge, build high-quality brands, form brand advantages, perfect consumer utility by dint of brand effect, and ensure sufficient profits for routine operation and development of enterprises after pricing rights are transferred to consumers. In every part, enterprises shall also utilize the advanced big data technology to collect first-hand materials, research into consumers' preferences and the market tendency, know about customers' comments and make further improvement for the purpose of gaining an advantage in the fierce market competition. For instance, Himalaya FM divides its contents into more than thirty sections and provides consumers with multiple custom channels. In addition, it also cooperates with several hardware manufacturers to create demands on special scenes and utilizes complementary advantage of the brands to extend their influence, thus successfully attracting more users and uplifting the user conversion rate.

Enterprises shall focus on their corporate culture, stimulate employees' creative thinking, refer to the knowledge economy character of the Internet age and the successful measures of those representative enterprises, provide new definition for the traditional 4Ps marketing theory, and expand the marketing channel from multiple angles, thereby attaining a favorable position in the market with less barriers to entry but more intense competition or creating new user demands.

Therefore, enterprises shall internally strengthen their construction, enhance their attractiveness to employees, and attach great importance to recruitment, training, motivation and etc; externally they shall attract consumers with better promotions, highlight the epochal character of product, price, channel and publicity, adopt strategies according to the oriented objects, and fulfill the unification of 
corporate culture and value.

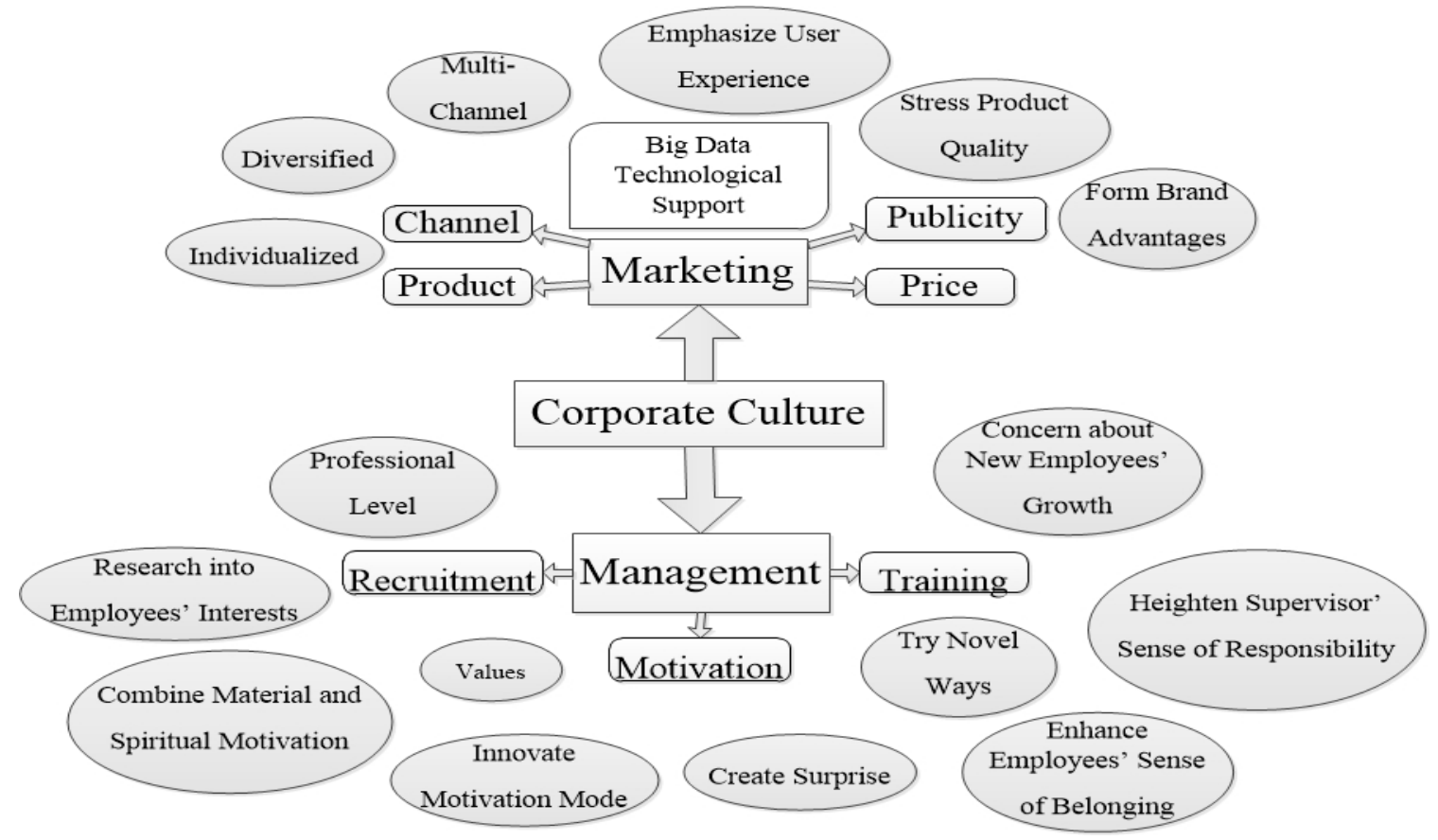

\section{Conclusion}

In the knowledge economy age, products enjoy a variety of forms, innovative products emerge endlessly, the way of pricing proceeds with significant reforms, and consumers gain their rights little by little. To adapt to the market and seize the chance, enterprises actively shall reform management mode, increase employees' happiness at work, enhance productivity, stimulate employees' creativity maximally and make full use of their own advantages. Meanwhile, they shall also make adjustment from multiple aspects, transmit product information in the way preferred by target groups, and uplift consumers' satisfaction.

However, as measurement indicators and appraisal systems for the quality of services offered by the knowledge service industry are not yet developed by the academic circle, it is hard to strengthen the supervision and effectively protect intellectual property rights, especially the live broadcast sector which involves both good and bad contents. The lack of empirical research on current innovation models and their efficiency makes it difficult for enterprises to compare with and learn from each other and then improve their own management models. Therefore, experts are expected to pay more attention to the quality assessment and performance of different services and carry out relevant researches.

\section{References}

[1] China Internet Network Information Center: The 40th China Statistical Report on Internet Development (2017)

[2] Chen Shaohua, The innovation, development, and model selection of knowledge service in the era of big data, J. Special Planning. 14 (2017) 6-9.

[3] The Organization for Economic Co-operation and Development: The knowledge-based economy (2017)

[4] Zhang Chunxiao, A study on the pricing mechanism of knowledge payment platforms based on the two-sided market theory, J. Journal of Jilin Business and Technology College. 4 (2017) 32-35.

[5] LIU Bing, Wang Xinyu, A survey of tipping on new media platforms, J. Youth Journalist. 22 (2017) 23-24. 
[6] Jiang Shimei, MENG Jing, Zhang Yuyao, Li Xiaochun, LIU Fang, Mode of knowledge worker management -Case study of Huawei, J. Technology Economics. 5 (2017) 43-50.

[7] Geng Tiancheng, Li Pengbo, LIANG Han, Gamification management of new generation staff from the perspective of internal and external motivation -Case study of Luogic Show, J. Management Review. 6 (2017) 108-115.

[8] LIU Yang, A discussion on marketing management in the age of knowledge economy, J. China Management Informationization. 16 (2017) 59-60.

[9] Zhang Zhenming, Research into the way of enterprise management innovation under the condition of knowledge economy, J. Business Management Space. 3 (2015) 15-17.

[10] Gu Jiqiang, Enterprise brand communication of knowledge quiz platforms on the Internet-case study of “Zhihu”, J. Journalism and Communication. 15 (2017) 32-33.

[11] Jiang Moya, Research into the development of mobile network broadcasting stations in the new media age-case study of Himalaya FM, J. A Vast View on Publishing. 14 (2017) 69-71. 